This item was submitted to Loughborough's Research Repository by the author.

Items in Figshare are protected by copyright, with all rights reserved, unless otherwise indicated.

\title{
The worked example effect, the generation effect, and element interactivity
}

\section{PLEASE CITE THE PUBLISHED VERSION}

https://doi.org/10.1037/edu0000018

\section{PUBLISHER}

American Psychological Association (APA)

\section{VERSION}

AM (Accepted Manuscript)

\section{PUBLISHER STATEMENT}

CAmerican Psychological Association, 2015. This paper is not the copy of record and may not exactly replicate the authoritative document published in the APA journal. Please do not copy or cite without author's permission. The final article is available, upon publication, at: https://doi.org/10.1037/edu0000018.

\section{LICENCE}

All Rights Reserved

\section{REPOSITORY RECORD}

Chen, Ouhao, Slava Kalyuga, and John Sweller. 2015. "The Worked Example Effect, the Generation Effect, and Element Interactivity”. figshare. https://hdl.handle.net/2134/12053721.v1. 


\title{
The Worked Example Effect, the Generation Effect, and Element Interactivity
}

Submitted date: $26 / 05 / 2014$

\section{Revision Submitted date: 15/11/2014}

\begin{abstract}
The worked example effect indicates that examples providing full guidance on how to solve a problem result in better test performance than a problem-solving condition with no guidance. The generation effect occurs when learners generating responses demonstrate better test performance than learners in a presentation condition that provides an answer. This contradiction may be resolved by the suggestion that the worked example effect occurs for complex, high element interactivity materials that impose a heavy working memory load whereas the generation effect is applicable for low element interactivity materials. Two experiments tested this hypothesis in the area of geometry instruction using students with different levels of prior knowledge in geometry. The results of Experiment 1 indicated a worked example effect obtained for materials high in element interactivity and a generation effect for materials low in element interactivity. As levels of expertise increased in Experiment 2, thus reducing effective complexity, this interaction was replaced by a generation effect for all materials. These results suggest that when students need to learn low element interactivity material, learning will be enhanced if they generate rather than study responses but if students need to learn high element interactivity material, study may be preferable to generating responses.

Keywords: Cognitive Load Theory, worked example effect, generation effect, element interactivity, guidance, expertise reversal effect.
\end{abstract}


The role of instructional guidance during teaching has been an important and controversial issue in instructional psychology (Ausubel, 1964; Craig, 1956; Kirschner, Sweller \& Clark, 2006; Mayer, 2004; Shulman \& Keisler, 1966). On the one hand, the direct instructional guidance that fully explains concepts and procedures aids learning, particularly for novel information (Kirschner et al., 2006). The worked example effect based on cognitive load theory presents one of the strongest data sets supporting this approach. Worked examples provide learners with full guidance that contains the key steps needed to solve a problem. Many studies since Sweller and Cooper (1985) have indicated that worked examples can result in better learning of solution procedures than practicing conventional problem solving with no guidance. On the other hand, there has been considerable interest in the design of constructivist learning environments within the framework of Problem-Based Learning (PBL), experiential learning, or inquiry learning that often provide minimal guidance to facilitate knowledge construction by learners (e.g., Kazemi \& Ghoraishi, 2012; Steffe \& Gale, 1995). The generation effect is often referenced in support of this approach. The generation effect describes a phenomenon that occurs when items (e.g., word lists) that are generated by learners in the presence of a stimulus and an encoding rule are better remembered than the same items that are simply read by learners (e.g., McElroy \& Slamecka, 1982; Slamecka \& Graf, 1978). The effect is regarded as evidence that active participation in the learning process produces better retention than passive observations. For example, in relation to $\mathrm{PBL}$, the generation effect has been interpreted as indicating benefits for learners in being actively engaged with problem solving cases allowing them to build their own understanding under the guidance of an instructor. It has been argued that the instructor should not build understanding for the students (Lai \& Tang, 1999).

Thus, these two effects have an apparent contradiction with the worked example effect implying more guidance should be provided to learners, whereas the generation effect 
suggests less guidance should be provided. The experiments reported in this article were based on the assumption that this contradiction could be resolved by considering the complexity of the learning materials used to demonstrate the effects. The worked example effect may require complex materials with the generation effect requiring much simpler materials. In cognitive load theory, the complexity of learning materials is described in terms of the concept of element interactivity.

\section{Cognitive load theory and element interactivity}

Cognitive load theory is an instructional theory that is based on some of the key characteristics of human cognitive architecture. In turn, that cognitive architecture is based on the information processing structures of evolutionary biology (Sweller, Ayres, \& Kalyuga, 2011; Sweller \& Sweller, 2006). Human cognitive architecture refers to the way in which the components, such as working memory and long-term memory, are organized. Aspects of that architecture relevant to instructional issues can be specified by five basic principles that underlie human cognitive architecture.

1. Information store principle. Human cognition relies on a large store of domainspecific information (Tricot \& Sweller, 2014) held in long-term memory (De Groot \& Gobet, 1996). Information is stored in the form of schemas (Chi, Glaser, \& Rees, 1982) in long-term memory. That knowledge is the primary source of activity and determines levels of expertise in a given area. Accordingly, a primary purpose of instruction is to increase the store of domain-specific knowledge held in long-term memory.

2. Borrowing and reorganizing principle. Given the immense size of the information store (Simon \& Gilmartin, 1973), efficient procedures for acquiring information are needed. The store is acquired primarily by borrowing information from other people by imitating what they do (Bandura, 1986), listening to what they say and reading what they write. Once borrowed, the information is usually restructured and organized for storage. 
3. Randomness as genesis principle. Whereas most information held in long-term memory is borrowed from other people, a mechanism for generating that information in the first instance is required. Information initially can be constructed by a random generation and test process during problem solving. If a solution to a problem is unavailable from one's own or another person's long-term memory via the borrowing and reorganizing principle, possible moves are randomly generated and then tested for effectiveness with successful moves retained in long-term memory and unsuccessful moves jettisoned.

4. Narrow limits of change principle. When solving novel problems using a random generate and test procedure, a mechanism is required to reduce combinatorial explosions. For example, if 3 elements are combined using the logic of permutations, there are $3 !=6$ possible permutations. For 10 elements there are $10 !=3,628,800$ permutations. Human cognition solves this problem by limiting the number of novel elements that working memory can deal with at any given time. Working memory has a limited capacity (e.g. Cowan, 2001; Miller, 1956) and a short duration (Peterson \& Peterson, 1959). These limits reduce the negative consequences of combinatorial explosions and reduce the possibility of large and rapid changes to long-term memory compromising the functionality of important stored information.

5. Environmental organizing and linking principle. This principle provides the ultimate justification for the human cognitive system. The limitations of working memory only apply to novel information held in working memory. They are irrelevant to organized, previously learned information retrieved from long-term memory (Ericsson \& Kintsch, 1995). Following appropriate signals from the environment, working memory can retrieve huge amounts of information from long-term memory and use that information to determine appropriate action. Ericsson and Kintsch coined the term "long-term working memory" to indicate the vastly different characteristics of working memory when it deals with novel 
information from the environment as opposed to stored information from long-term memory. Working memory is able to handle large amounts of stored, organized information from longterm memory easily and rapidly with no known capacity or temporal limitations.

The cognitive load imposed by novel information can be divided into intrinsic and extraneous cognitive load (Sweller, 2010; Sweller et al., 2011). Intrinsic cognitive load is caused by learner cognitive activities required to achieve a learning goal. It is determined by the levels of element connectedness that depends on the nature of information and the learners' prior knowledge level. This load is directly relevant to learning the essential structure of information (Sweller, 1994).

Element interactivity is an index of the complexity of learning material within the framework of cognitive load theory. That index depends heavily on the cognitive architecture outlined above. A usable index of complexity cannot simply refer to the characteristics of the information being considered. It simultaneously must consider the knowledge held in longterm memory. For example, the set of symbols "cat" may be immensely complex for someone beginning to learn to read English with no background using the Latin alphabet but simple for anyone reading this article. The environmental organizing and linking principle explains why information held in long-term memory can render "cat" simple and easy to deal with because it can be treated as a single element whereas the absence of that information from long-term memory can render the same information impossible to deal with because it consists of multiple elements that may overwhelm working memory. Any usable index of complexity must take these characteristics of human cognition into account.

Of course, whereas element interactivity depends heavily on learner expertise, it depends equally heavily on the nature of the information. Some learning materials can be processed individually, such as learning English words for second language learners or chemical symbols in the periodic table. These materials can be learned independently and 
without referring to other content, so the number of interactive elements for this kind of material is 1 rendering this information low in element interactivity. However, if the materials cannot be learned independently but must be processed simultaneously in working memory, the number of interactive elements will be increased rendering the material relatively high in element interactivity. An example is learning to solve a problem such as: $x+5=8$, solve for $x$. In order to solve this problem, novice learners should first hold a series of single mathematics elements ( $\operatorname{such}$ as $x, 5,8,+,=$ ) in their limited working memory, totaling 5 interactive elements. If they only hold these single mathematics symbols, they cannot solve this problem. The learners also need to process the relations between different symbols in order to understand this equation and to find the value of $x$. They need to note that 5 is added to $x$, that subtracting 5 will eliminate the addend, that if 5 is subtracted from one side of an equation it also must be subtracted from the other side, and lastly, that subtracting 5 from both sides will solve the problem. Therefore, the total number of interactive elements for this material is approximately 9 . For this simple algebra problem, novice learners may need to process all of those interactive elements simultaneously in working memory, resulting in a high-element interactivity task.

Of course, with sufficient expertise, the number of interacting elements of this algebra problem would reduce to 1 because an expert can retrieve the entire solution from long-term memory as a single schema. This task is high in element interactivity only for novice learners who are using the randomness as genesis principle to solve the problem. As they have not formed relevant schemas for this equation, they have to generate moves randomly and test them for effectiveness (e.g., by using a trial-and-error technique). For more knowledgeable learners, the interacting elements are incorporated in the relevant schemas held in long-term memory. Using the information store and the environmental organizing and linking principles, experienced learners can retrieve the relevant schema from long-term 
memory directly to solve this equation, treating the whole equation as a single familiar element (chunk) of information to minimize working memory resources required for processing this information. The schema, stored in long-term memory, is transferred to working memory and used to solve the above equation. In this way, learning changes the element interactivity and intrinsic cognitive load of information.

Thus, the complexity of materials and levels of element interactivity are always related to levels of learner prior knowledge (or expertise) in a task domain. Element interactivity levels can be estimated by the number of interacting elements incorporated in learning materials for specific learners (Sweller \& Chandler, 1994; Tindall-Ford, Chandler \& Sweller, 1997) and will change for other learners with differing levels of expertise. If materials have a low level of element interactivity and so a low intrinsic cognitive load, the instructional designs used may not interfere with students' learning, resulting in the element interactivity effect of cognitive load theory (Sweller et al., 2011). Therefore, all cognitive load effects tend to be obtained only with materials that are characterized by high levels of element interactivity and so a high intrinsic load (Sweller, 2010; Sweller \& Chandler, 1994).

Based on the above analysis, interacting elements can be defined as elements of information that must be processed simultaneously in working memory to achieve understanding because they are logically related (Sweller, 2010; Sweller et al., 2011) but that have not as yet been integrated and stored in long-term memory as a single chunk or schema. The estimated number of interactive elements that must be dealt with by a given individual or group of individuals performing a particular task or learning a particular concept or procedure can be counted. The total number of interactive elements indicates the level of element interactivity of this material for given learners.

In contrast to intrinsic cognitive load, extraneous cognitive load can be altered by instructional interventions, as this load is influenced by the way instructional materials are 
designed and presented (van Merriënboer \& Sweller, 2005). Element interactivity also is central to extraneous cognitive load. Whereas interacting elements that are an unavoidable part of a task determine intrinsic cognitive load, interacting elements that are introduced solely because of the manner in which instruction is designed determine extraneous cognitive load (Sweller, 2010). This load is imposed by suboptimal teaching methods and should be reduced or eliminated to increase working memory resources available to deal with intrinsic load (often referred to as germane cognitive load) and so enhance learning. The resources of working memory allocated to deal with intrinsic load (germane resources) need to be maximized, whereas resources allocated to deal with extraneous load that is irrelevant to learning should be minimized. The worked example effect, discussed next, is a major cognitive load effect caused by an excessive extraneous cognitive load when problem solving is used as an instructional method.

\section{Worked example effect}

According to Cognitive Load Theory, worked examples that provide full guidance to learners on how to solve a problem can result in better performance than a problem solving condition that has no guidance, resulting in the worked example effect (Cooper \& Sweller, 1987; Sweller \& Cooper, 1985). The theoretical rationale for the worked example effect flows directly from the human cognitive architecture outlined above (Sweller et al., 2011).

According to the information store principle, learning consists of the acquisition of large amounts of domain specific knowledge in long-term memory (De Groot \& Gobet, 1996; Tricot \& Sweller, 2014). Just as De Groot demonstrated that chess expertise consists of knowledge of problem states and the best moves associated with each state, knowledge of academic disciplines should equally consist of knowledge of problems states and the moves associated with those states. Worked examples provide that knowledge directly. From the borrowing and reorganizing principle, the required knowledge is best obtained from other 
people, and worked examples provide knowledge from other people. In contrast, problem solving, with its emphasis on the randomness as genesis principle, only should be used when knowledge from others is not available because it imposes a heavy working memory load in accord with the narrow limits of change principle. Evidence for the heavy working memory load associated with problem solving comes from computational models (Sweller, 1988) and subjective ratings of difficulty (Paas, 1992). Once knowledge is available in long-term memory, it can be used to efficiently solve problems using the environmental organizing and linking principle. Because domain-specific knowledge is acquired more efficiently using worked examples than problems, a comparison of studying worked examples rather than solving problems can be expected to demonstrate the worked example effect. That effect has been demonstrated repeatedly in many experiments (Atkinson, Derry, Renkl, \& Wortham, 2000; Renk1, 1997; Schwonke et al., 2009; Sweller et al., 2011).

There are a variety of documented conditions under which the worked example effect, like all cognitive load effects, will not be obtained (Sweller et al., 2011). One of those conditions concerns levels of element interactivity. The worked example effect should only be obtained if element interactivity is high resulting in a high intrinsic cognitive load. If intrinsic cognitive load is high, extraneous cognitive load should be controlled by using worked examples instead of problem solving. Therefore, a comparison of worked examples with problem solving results in the worked example effect only under high element interactivity conditions.

However, if materials are low in element interactivity with the intrinsic cognitive load of materials low, instructional procedures associated with cognitive load theory such as procedures based on the worked example effect, no longer apply. Controlling extraneous cognitive load is unnecessary when the intrinsic cognitive load of the materials used is low because the total cognitive load may not exceed working memory limits. The possible 
occurrence of cognitive load effects such as the worked example effect under high but not low element interactivity conditions would provide an instance of the element interactivity effect.

As indicated above, element interactivity does not just depend on the characteristics of the materials. It also depends on the knowledge base of learners. High element interactivity material for novices may be low element interactivity material for more knowledgeable learners. Accordingly, as levels of expertise increase, we might expect the worked example effect to reduce and eventually reverse. Precisely this effect has been obtained (Kalyuga, Chandler, Tuovinen, \& Sweller, 2001) providing an example of the expertise reversal effect (Kalyuga, Ayres, Chandler, \& Sweller, 2003). An interpretation of this finding that accords with the present theoretical framework is that increased expertise reduces element interactivity and with reduced element interactivity, a result similar to the generation effect appears.

\section{Generation effect}

In marked contrast to the worked example effect, the generation effect occurs when learners who generate responses themselves perform better than those who study presented answers to questions (Slamecka \& Fevreiski, 1983; Slamecka \& Graf, 1978). This phenomenon is robust for various situations and different kinds of memory tests such as cued, free recall and recognition tests. The traditional format in studies of the generation effect is to use word pairs that include a stimulus as the cue and the first letter of the target word, with a rule indicating how the response is to be generated, for example, COLD-H_(OPPOSITE). Irrespective of specific formats, the generation effect has been found in many studies with generation conditions resulting in better memory traces than presentation conditions.

The majority of the studies have used highly familiar, low element interactivity materials that are unlikely to have imposed a heavy working memory load. As examples, 
Slamecka and Graf (1978) used regular word pairs with five rules (associate, category, opposite, synonym and rhyme) as cues to obtain the generation effect using free recall, cued recall and recognition tests. Each paired associate can be learned without reference to any other pair and so is low in element interactivity. McFarland, Frey and Rhodes (1980) obtained the generation effect with sentences. They asked participants to fill in a missing word to generate meaningful sentences. Schemas for sentences ensure that element interactivity is low. McNamara and Healy (2000) used arithmetic problem solving tasks with one group of undergraduate learners required to calculate the answers to multiplication problems whereas another group was presented the answers. Participants then were required to recall the answers to the problems. The group that generated the answers was better able to remember them than the group presented the answers. In this experiment, McNamara and Healy used problem solving tasks but the posttest consisted of a simple memory task requiring recall of low element interactivity material. Most, possibly all of the literature on the generation effect used material that imposed a low working memory load due to low element interactivity.

Several hypotheses have been proposed to explain the generation effect (Bertsch, Pesta, Wiscott, \& McDaniel, 2007). Cognitive effort is one factor that may affect the generation effect (Slamecka \& Fevreiski, 1983). Griffith (1976) and McFarland, Frey and Rhodes (1980) suggested that the generation effect was attributable to greater amounts of cognitive effort required by generation compared to presentation of information. If the goal of processing in generation is to make a stimulus cognitively identifiable, reaching that goal is more effortful (Tyler, Hertel, McCallum, \& Ellis, 1979) as it requires more precise discrimination of stimuli (Jacoby, Craik, \& Begg, 1979). Therefore, it demands more elaboration of stimuli (Craik \& Tulving, 1975) than does reading and that increased effort may be important in the generation 
effect. Based on their meta-analysis, Bertsch et al. (2007) suggested there is some evidence for this hypothesis.

The selective rehearsal displacement hypothesis also may account for the generation effect (Slamecka \& Katsaiti, 1987). It suggests that under mixed generation and presentation conditions, learners may spend more time on the generation than the presentation items resulting in a generation effect. Of course, this hypothesis cannot explain the generation effect using between-subjects designs so at best, it is a partial explanation. Bertsch et al. (2007) also merged several hypotheses under transfer appropriate processing according to which the more the processes used during learning overlap with those used during the test phase, the better the test performance would be. In general terms, learners required to generate responses during both learning and test phases should perform at a higher level on test than learners who read responses when learning but generate responses in the test. Based on their meta-analysis, the evidence supporting these hypotheses is mixed.

All of these hypotheses can, at least in part, explain and contribute to the findings on the generation effect. None of them can explain (or are intended to explain) the worked example effect, the results of which are contrary to those expected according to the generation effect. Indeed, all of these hypotheses are directly contradicted by the worked example effect. Solving problems is inferior to studying worked examples but requires more effort than studying worked examples (Paas, 1992; Paas \& van Merrienboer, 1994), contradicting the cognitive effort hypothesis; takes longer than studying worked examples (Cooper \& Sweller, 1987; Sweller \& Cooper, 1985), contradicting any hypothesis based on generation taking more time than presentation; and requires less disparity between learning and test conditions than studying worked examples, contradicting the transfer appropriate processing hypothesis. 
The hypotheses used to explain the generation effect cannot be used to explain the worked example effect. Of course, the explanation of the worked example effect, that the guidance provided to assist learners in solving problems reduces working memory load thus facilitating learning is equally inapplicable to the generation effect or, indeed, the reverse worked example effect (Kalyuga et al., 2001). Different hypotheses are needed to explain those contrary results. Those hypotheses suggest that the categories of material used to demonstrate the worked example and generation effects are different, potentially resolving the contradiction.

\section{Present study}

According to the above overview, the contradiction between the worked example and generation effects may be resolved by considering element interactivity as a critical factor. The worked example effect (i.e., the superiority of high levels of guidance) may occur for high element interactivity materials whereas the generation effect (i.e., the superiority of low levels of guidance) may be applicable for low element interactivity materials. Accordingly, it was hypothesized that the specific type of effect could be predicted by considering the levels of element interactivity of the corresponding materials. This hypothesis was tested using geometry learning materials that differed in element interactivity.

The specific hypotheses were as follows:

1. A dis-ordinal interaction of levels of guidance and element interactivity will be obtained. High levels of guidance may be superior to low levels of guidance using materials high in element interactivity resulting in the worked example effect, whereas low guidance may be superior to high guidance using materials low in element interactivity resulting in the generation effect. As indicated above, complex material may need explicit guidance to assist learners to understand the material. Simpler material may not require explicit guidance. 
2. At higher levels of learner expertise, the interaction of guidance and element interactivity may disappear because higher levels of expertise should reduce element interactivity. High guidance may become inferior to low guidance with materials that are high in element interactivity for novices but low in element interactivity for more expert learners (i.e., the worked example effect will disappear), whereas low guidance may remain superior to high guidance with low element interactivity materials demonstrating the generation effect. Thus, for learners with high levels of prior knowledge, the generation effect was hypothesized to be obtained for all materials with the interaction of guidance and element interactivity disappearing.

Two experiments were conducted to test these hypotheses in the area of geometry instruction with students at two different levels of prior knowledge in geometry. A 2 (guidance: high and low) x 2 (element interactivity: high and low) experimental design was used in both experiments (see Figure 1 for the general experimental design used). Learners with low levels of prior knowledge were used in Experiment 1 and so the dis-ordinal interaction of Hypothesis 1 was relevant whereas learners with higher levels of knowledge were used in Experiment 2 and so the interaction was hypothesized to reduce or disappear.

\section{Experiment 1}

The purpose of this experiment was to investigate the hypothesis of a dis-ordinal interaction between levels of guidance and levels of element interactivity using Year 4, primary school learners studying geometry topics that were either high or low in element interactivity for these students. As indicated above, according to the element interactivity effect, if learning materials are low in element interactivity, then other cognitive load effects such as the worked example effect are unlikely to be obtained. Therefore, in this experiment, high-element interactivity materials were used to test for the worked example effect by comparing studying worked examples (high guidance) with problem solving (low guidance). 
Low- element interactivity materials were used to test for the generation effect by presenting learners with answers to memory questions (high guidance) or having them generate answers themselves (low guidance).

\section{Method}

Participants. The participants were 41 Year 4 students from a primary school in Chengdu, China. They were approximately 10 years old. They were randomly assigned to either the generation or presentation group in the first phase of the experiment and then half of the students from the generation group were randomly assigned to the problem solving group and the other half to the worked example group in the second phase. Similarly, half of the students from the presentation group were allocated randomly into either the worked example or problem solving group. Four students in the generation group and four in the presentation group did not complete the entire procedure. These eight students were eliminated from the data analyses testing for both the generation and worked example effects, leaving 33 students. In class, all students had only studied formulae for the area and perimeter of squares and rectangles and so were regarded as novices with respect to other formulae used in this study to test for the generation effect. Similarly, none of the students had been taught to solve the problems used to test for the worked example effect.

Materials. To test for the generation effect, 11 geometry formulae were chosen from textbooks used in primary and secondary schools. There were three surface area formulae, four perimeter formulae and four area formulae (see Appendix 1 for examples of the geometry formulae). In order to allow the Year 4 students who had not as yet studied algebra understand the formulae, the presentation avoided algebraic expressions such as "ab" which were replaced by a $\mathrm{x} b$.

Levels of element interactivity were estimated for all materials using the method illustrated by the following examples. Assume students are asked to remember the formula 
used to calculate the area of a parallelogram, a task used to test for the generation effect (Appendix 1). Based on the concept of element interactivity, Year 4 students needed to memorize 5 elements of the equation, Area $=l x h$. Because there are 5 elements, they do not interact and neither do they need to be connected to the diagram. Each can be memorized separately and if one is forgotten, it does not affect any of the others. For example, if a pronumeral is forgotten and replaced by a different symbol, it has no effect on any of the other symbols. Therefore, this material required learners to deal with only 1 element at a time and so the interacting element count is 1 .

In contrast, assume students are asked to calculate the area of a composite shape, a task used to test for the worked example effect (see Appendix 2-1 for an example of the task). Year 4 students who had not learned previously how to calculate the area of composite shapes needed, first, to identify the four equal length lines, including the missing line, FC, that form a rhombus. They then needed to identify the four lines that constitute a trapezium, again including the missing line, FC. Next, they had to determine that they needed to calculate the areas of both requiring another two elements. To actually calculate the two areas, students needed to know the meaning of $a$ and $b$ in the rhombus and the multiplication relationship between them, as well as the meaning of $a, b$, and $h$ in the trapezium and addition, multiplication, and division by 2 , that together involved 9 elements. Finally, adding together those two separate area values involved another element. Therefore, in total, it can be estimated that about 20 interacting elements were involved in this task rendering it high in element interactivity for Year 4 students, compared to the material required to memorize the area of a parallelogram.

Students were given three booklets printed on A3 paper. The first booklet contained eleven basic geometry formulae (Appendix 1) and was common to both conditions. All of the perimeter formulae were presented first followed by all of the area formulae and then all of 
the surface area formulae. The second booklet differed for the generation and presentation groups. For the presentation group, the content was identical to the first booklet, whereas for the generation group, only the names of each of eleven formulae and their relevant geometric shapes were included as students needed to generate each formula by themselves. The third booklet (common to both conditions) was blank to allow students to write out their answers in the free recall test. They were required to write out as many of the formulae they had studied from the first and second booklets.

To test for the worked example effect, students again were given three booklets. The first booklet was identical to the first booklet used to test for the generation effect. Its function was revision or review of the previously learned information. The second booklet differed for the worked example and problem solving groups. The booklet for the worked example group contained two worked examples each followed by a similar problem for students to solve (Appendix 2). Students in the problem solving group were required to solve the same 4 problems by themselves with no worked examples provided. The third booklet contained five test problems for students to solve. The first three problems required learners to calculate the area of a composite shape and the other two required students to calculate the area of shaded sections of the diagrams (Appendix 3). All booklets had a cover page identical to the one for the generation effect.

Procedure. The generation effect phase of the experiment lasted for one class period of 35 minutes. The procedure for this phase of the experiment is indicated in Figure 2.

Prior to studying the first booklet, students were re-seated according to the group into which they were randomly placed (7 minutes).

The study stage (10 minutes). After being re-seated, students began studying the first booklet. They could make notes in this booklet if they needed to. After 10 minutes, all students handed in this booklet. 
The generation or presentation stage (10 minutes). The experimenter distributed the second booklet to students in the generation and presentation groups separately. Students in the generation group were required to generate all of the formulae they had studied in the first booklet, whereas students in the presentation group were required to again study those formulae. No one could hand in this booklet before 10 minutes had elapsed. Any students who completed their task in less than 10 minutes were told to review the material again. After 10 minutes, all students handed in this booklet.

The free recall test stage ( 8 minutes). The test required students to write out as many of the formulae that they had studied in the first and second booklets. Students could only hand in their test booklet after 8 minutes had elapsed. Therefore, if students finished early, they were required to review their answers. When scoring the test, a correct formula was awarded 1 mark. Therefore, the maximum score in the free recall test was 11. Each student's score out of 11 was converted into a percentage score for analysis providing the scores testing knowledge of the low-element interactivity material (see Table 1 for the relevant mean percentages).

The worked example effect phase of the experiment also lasted for one class period of 35 minutes that occurred 4 hours later on the same day after the generation effect phase. The procedure for this phase of the experiment is diagrammed in Figure 3. Prior to the experiment, the students already had been randomly chosen from the generation and presentation groups to form the worked example and problem solving groups. Students were reseated according to the group to which they had been allocated (7 minutes).

The study stage (10 minutes): The procedure for this stage was identical to that used for the equivalent stage in the generation effect phase of the experiment.

The worked-example or problem-solving stage (10 minutes). The general procedure was identical to that used in the generation effect phase. Students in the worked example 
group were required to first study the worked example of Problem 1 indicating how to calculate the area of a composite shape and then to solve a similar problem (Problem 2). A similar procedure was followed for Problem 3 (a worked example) and Problem 4 (a similar problem to Problem 3 that students had to solve themselves rather than study as a worked example). Students in the problem solving group were required to solve the same four problems (Problems 1 - 4) used in the worked example group by themselves, with none of the problems presented as worked examples.

The test stage (8 minutes). Again, the general procedure was identical to that used in the generation effect phase. The test required students to solve 5 problems (see Appendix 3 for examples of test problems). Students could obtain a maximum of 4 marks on each of the first three problems ( 1 mark for constructing the line to divide the composite shape into two basic geometry shapes; 1 for correctly using the area formula of one of the basic geometry shapes; 1 for correctly using the area formula of the other basic geometry shape; 1 for adding the two areas). The maximum score for both of the last two problems was also 4 ( 1 for calculating the area of the whole shape; 1 for correctly using the area formula of one of the basic geometry shapes; 1 for correctly using the area formula of the other basic geometry shape; 1 for subtracting the area of the non-shaded parts from the total area). Each student's total score out of 20 ( 5 problems each with a maximum score of 4 ) was converted to a percentage score for analysis. The internal reliability of this test using Cronbach's $\alpha$ was .72 after deleting the $3^{\text {rd }}$ test question to increase the reliability of the test. These scores provided the dependent variable testing for knowledge of the high-element interactivity material (see Table 1 for the mean percentage test scores).

\section{Results and Discussion}

Means and standard deviations of percentage test score results may be found in Table 1. These results were analyzed using a 2 (levels of guidance) x 2 (levels of element interactivity) 
ANOVA with repeated measures on the element interactivity factor. All means, standard deviations and analyses were based on the 4 test questions remaining after eliminating Question 3, but it should be noted that the patterns of significance were identical to those obtained using all 5 test questions.

The main effect of guidance was not significant, $F(1,31)=.002$, MSe $=241.94, p$ $=.964, \eta_{p^{2}}=0$. The main effect of element interactivity was significant, $F(1,31)=19.85$, MSe $=281.86, p<.001$, Wilks' Lambda $=.610, \eta_{p^{2}}=.390$. The low element interactivity material percentage correct test scores were higher than the high element interactivity test scores. The interaction between guidance and element interactivity was of primary interest in this experiment and was significant, $F(1,31)=9.98, \mathrm{MSe}=281.86, p=.004$, Wilks' Lambda $=.756, \eta_{p^{2}}=.244$.

Following the significant interaction, simple effects tests were conducted. For the low element interactivity material testing for the generation effect, the effect of guidance was significant, $\mathrm{t}(31)=-3.08, \mathrm{SE}_{\mathrm{diff}}=4.30, p=.002(1$-tailed $), d=.96$. The mean percentage correct scores indicated that low guidance was superior to high guidance demonstrating a generation effect.

For the high element interactivity material testing for the worked example effect, the effect of guidance also was significant, $\mathrm{t}(31)=2.09, \mathrm{SE}_{\mathrm{diff}}=6.28, p=.02$ (1- tailed), $d=.65$. The mean percentage correct scores indicated that high guidance was superior to low guidance demonstrating a worked example effect.

In Experiment 1, it was hypothesized that an interaction of guidance and element interactivity would be obtained. High guidance was predicted to be superior to low guidance using materials high in element interactivity, whereas low guidance was predicted to be superior to high guidance with materials low in element interactivity. The results of Experiment 1 confirmed this hypothesis with a dis-ordinal interaction of guidance and 
element interactivity obtained. The simple effect tests indicated that students who generated formulae during a study stage better memorized those formulae than students presented the formulae, in line with the generation effect. For materials high in element interactivity, students who studied worked example-problem pairs were better at solving test problems than students who only solved problems by themselves during the study stage, in line with the worked example effect.

\section{Experiment 2}

Experiment 2 again tested for an interaction between guidance and element interactivity with older, more expert learners using similar materials to those of Experiment 1. It was hypothesized that the interaction should be reduced or eliminated using students who had a reduced requirement for worked examples. The generation effect should be obtainable with more knowledgeable students but with increased expertise, the worked example effect should be reduced, eliminated or even reversed due to the expertise reversal effect. This effect occurs when one instructional procedure that is better than another for novices loses its relative advantage as expertise increases. For example, as found in Experiment 1, the use of worked examples rather than problem solving may be beneficial for novice learners. With increasing expertise, the advantage of worked examples may decrease or even reverse to a disadvantage because with increasing expertise, studying worked examples becomes a redundant activity. Increases in expertise should have the same effect as decreases in element interactivity. In Experiment 2, by increasing the expertise of the learners, all of the materials should be effectively low in element interactivity for these relatively knowledgeable students.

The general procedure was similar to that used in Experiment 1. Low element interactivity materials were used to test for the generation effect by presenting learners with answers to memory questions (high guidance) or having them generate answers themselves (low guidance), whereas higher-element interactivity materials were used to test for the 
worked example effect by comparing studying worked examples (high guidance) with problem solving (low guidance). The same two phases of Experiment 1 also were used in Experiment 2. Students first were presented the low element interactivity materials to test for the generation effect followed by the high element interactivity materials to test for the worked example effect.

\section{Method}

Participants. The participants were 38 Year 7 students, from a secondary school in Chengdu, China. They were approximately 13 years old. They were randomly assigned to groups using the procedure of Experiment 1. Two students in this experiment did not complete the entire procedure. These two students were eliminated from the data analysis for both the generation and worked example effect phases, leaving 36 students. In class, all students previously had studied the area and perimeter formulae used in this study to test for the generation effect. Similarly, all students had been taught to solve the problems used to test for the worked example effect approximately a year previously. Therefore, Year 7 students were regarded as relatively expert with respect to the formulae as well as the problems used in Experiment 2.

Materials. To test for the generation effect in this experiment, the same materials that had been used to test for the generation effect in Experiment 1 were used, except that all formulae were in algebraic form. However, the materials used to test the worked example effect in Experiment 1 were changed in Experiment 2 (Appendix 4). In this experiment, the problems used in the second booklet that divided students into the worked example and problem-solving groups retained the first two problems that were concerned with calculating the area of a composite shape used in Experiment 1. The last two problems were substituted by the three similar problems used in the test phase of Experiment 1 (Questions 1, 2 and 3). Therefore, the test questions for the worked example effect used in the third booklet also had 
to be changed in this experiment. Only the first two test questions used in the test of Experiment 1 were used in the Experiment 2 test, with all of the test questions requiring a calculation of the area of a composite shape. Therefore, this test was different from the corresponding test used in Experiment 1 (see Appendix 5 for an example of a test problem). In order to facilitate marking, only one mathematics topic was tested: calculation of the area of a composite shape.

When counting the number of interacting elements to evaluate the effective level of element interactivity in instructional materials of this experiment, the relatively higher level of learner expertise was taken into account. For example, for the area of a parallelogram formula in the material for testing the generation effect (used above in Experiment 1 to demonstrate the procedure), it was considered that Year 7 students had acquired the relevant knowledge, so the relevant schema that combined the single elements of this formula was already available in their long-term memory. Therefore, they did not need to consider the meaning of $l$ and $h$ separately, and the relation among $l, h$ and the area formula. They could just use the stored schema to deal with this memorization task. Therefore, the number of interacting elements for Year 7 students should be 1 because the relevant schema acts as a single entity to be processed in working memory.

A similar reduction of the number of interacting elements applied to material used for testing the worked example effect. For example, in the case of the task used above in Experiment 1 to illustrate the procedure, Year 7 students are likely to have already acquired the relevant schemas for perceiving and calculating the areas of a rhombus and a trapezium from their long-term memory as single units to process in working memory, thus reducing the number of interacting elements to two. Combining the values of these two areas using the schema for composite shapes as an entity results in the total number of interacting elements for this problem for Year 7 students to be 3, that is a low level of element interactivity. 
Procedure. The general procedure was identical to Experiment 1.

Scoring. The scoring procedure for the generation effect was identical to that used in Experiment 1 . For the worked example effect, only the scoring method used to calculate the area of a composite shape was used. The internal reliability of the test for the worked example effect using Cronbach's $\alpha$ was .90 , after eliminating the $1^{\text {st }}$ test question to increase the reliability of the test.

\section{Results and Discussion}

Means and standard deviations of percentage correct test score results may be found in Table 2. These results were analyzed using a 2 (levels of guidance) x 2 (levels of element interactivity) ANOVA with repeated measures on the element interactivity factor. All means, standard deviations and analyses were based on the 4 test questions remaining after eliminating Question 1, but it should be noted that the patterns of significance were identical to those obtained using all 5 test questions.

The main effect of guidance was significant, $\mathrm{F}(1,34)=5.24, \mathrm{MSe}=605.98, p=.028$, $\eta_{\mathrm{p}^{2}}=.134$. The main effect of element interactivity was not significant, $\mathrm{F}(1,34)=.001$, MSe $=610.02, p=.971$. Wilks' Lambda $=1.00, \quad \eta_{p^{2}}=0$. The interaction of guidance and element interactivity was of primary interest in this experiment but was not significant, $\mathrm{F}(1$, 34) $=.00, p=.933$. Wilks' Lambda $=1.00, \eta_{\mathrm{p}^{2}}=0$.

It was hypothesized that when using older, more knowledgeable students in Experiment 2 , the interaction of guidance and element interactivity should be reduced compared to the previous experiment or eliminated. The worked example effect was predicted to be eliminated or reversed with increases in expertise thus reducing or eliminating the interaction. Results of this experiment supported this hypothesis with no interaction of guidance and element interactivity obtained. Increased guidance had a similar negative effect on both higher and lower element interactivity material. In other words, in contrast to Experiment 1 , 
the generation effect was obtained for both lower and higher element interactivity material with no sign of the worked example effect for the high element interactivity material.

\section{General Discussion}

We attempted to resolve the apparent contradiction between the generation effect and the worked example effect by hypothesizing that the generation effect would be more likely to be obtained using low element interactivity information whereas the worked example effect required high element interactivity information. Experiment 1 provided support for this hypothesis by demonstrating a dis-ordinal interaction of guidance and element interactivity with Year 4 students who were regarded as novices for the learning materials used. Specifically, for materials high in element interactivity, high guidance in the form of worked examples was superior to low guidance in the form of problems to solve, demonstrating the worked example effect. High guidance permits the borrowing and reorganizing principle to come into play because instructional information is provided to learners, allowing them to "borrow" information from instructors whereas low guidance requires the randomness as genesis principle to be used because learners must generate responses randomly if relevant information is not available to them. Borrowing information from others should reduce cognitive load compared to generating the information oneself. For materials low in element interactivity, low guidance in the form of learners generating formulae was superior to high guidance in the form of learners being presented the formulae indicating the generation effect. Reducing cognitive load by using the borrowing and reorganizing principle is unnecessary for low element interactivity material because the cognitive load is already low and indeed, based on the generation effect, attempts to reduce cognitive load are likely to be counterproductive.

The second hypothesis was that as levels of learner expertise increase, actual levels of element interactivity for these learners should decrease and so the interaction between element interactivity and guidance obtained in Experiment 1 should reduce or disappear to be 
replaced by superior performance by both the low guidance, generation and the problem solving groups over their high guidance controls represented by the presentation and worked examples groups. According to the environmental organizing and linking principle, with increased expertise, interacting elements should be incorporated into schemas held in longterm memory and so should no longer impose a heavy working memory load. If so, the worked example effect should no longer be obtainable.

Experiment 2 used similar material to that presented to Year 4 students in Experiment 1 but this time presented to Year 7 students. For Year 7 learners, the material constituted revision or review because they had studied the topic one year previously. The interaction of guidance and element interactivity disappeared with the increase in levels of expertise because the worked example effect reversed with the increase in expertise. The generation effect was still robust and was found for both sets of materials. In other words, low guidance was superior to high guidance for both sets of materials in Experiment 2.

A comparison of the worked example effect in Experiment 1 and its reversal in Experiment 2 provides a clear example of the expertise reversal effect. In Experiment 1, the worked example group was superior to the problem solving group, demonstrating the worked example effect. That result was reversed when using more knowledgeable students in Experiment 2. The worked examples were redundant for the more knowledgeable learners and so instead of obtaining a worked examples effect, we obtained a generation effect. With increases in expertise, most cognitive load effects first disappear and then reverse. In the case of the worked example effect, studying worked examples is superior to solving problems when testing novices but this difference disappears and then reverses with increases in expertise in the domain (Kalyuga et al., 2001). The contrasting results of Experiments 1 and 2 may be due entirely to the expertise reversal effect. 
Indeed, it may be plausible to suggest that the interaction between levels of guidance and element interactivity found in Experiment 1 is itself a form of the expertise reversal effect. According to cognitive load theory, changes in expertise result in changes in element interactivity as interacting elements are subsumed into knowledge held in long-term memory resulting in changes in effective working memory capacity limits. Material that is high in element interactivity for novice learners should be lower in element interactivity for relatively more knowledgeable learners. Instead of having to deal with large numbers of interacting elements via the narrow limits of change principle, many elements can be dealt with simultaneously using the environmental organizing and linking principle. In Experiment 1, the low element interactivity material that yielded the generation effect consisted of information that learners could easily learn. They had sufficient knowledge to be able to acquire the information readily by generating it rather than having it presented. They did not have sufficient information to easily generate the problem solutions of the high element interactivity information. When using participants who did have sufficient information to generate solutions readily in Experiment 2, the generation effect was obtained for both sets of material.

Since its inception, cognitive load theory has been applied largely, though not entirely, to novices for whom the material they were required to learn in a given area was complex and difficult due to the working memory load that was imposed. The theory was never intended to apply to information that was difficult for reasons other than a heavy working memory load. Having to learn a large number of elements that do not interact provides an example of an area that can be difficult for students for reasons other than a heavy working memory load. Once knowledge held in long-term memory renders information simple rather than complex, cognitive load theory has used the redundancy effect (Chandler \& Sweller, 1991) to explain why information should be generated rather than presented (Kalyuga et al., 2003). 
Unnecessarily processing redundant information may increase cognitive load. It is possible that unnecessarily reading presented information may be more cognitively demanding than generating that information oneself when the information is highly familiar. Nevertheless, any one or a combination of the reasons discussed in the introduction to this paper may provide suitable explanations of the generation effect.

A major limitation of the current study is that whereas the concept of cognitive load was used to hypothesize and explain the findings, we had no independent measure of cognitive load. Cognitive load usually is assessed using subjective ratings of mental effort or task difficulty (Paas \& van Merrienboer, 1993). It can be difficult to measure using young students tested under relatively standard, ecologically valid classroom conditions. Lee (2013) indicated that students younger than 15 years of age might not be suitable participants for using subjective ratings of cognitive load. As the participants used in the two experiments were 10-13 years in age, subjective rating of cognitive load may not be appropriate. In future studies, more mature participants might be used allowing a more ready use of subjective ratings of cognitive load.

We believe the current results have considerable importance from an instructional perspective. They suggest that when dealing with complex material that learners may have difficulty understanding, high levels of guidance are likely to result in enhanced performance over lower levels of guidance. In contrast, when dealing with simple material that is easy for students to understand either because there are few interacting elements or because previously high element interactivity material has been learned and incorporated into knowledge held in long-term memory, learners should practice generating responses rather than being shown them. Most curricula include both high and low element interactivity material. Based on the current study, learners should be encouraged to generate responses 
when dealing with low element interactivity material but should have complex, high element interactivity concepts and procedures explicitly demonstrated.

All instructional effects are likely to have limits with no effect occurring under all conceivable conditions. The generation and worked example effects are not exceptions. Interestingly, the conditions under which either effect can occur appear to provide the limits under which the other effect can be obtained.

\section{References}

Atkinson, R. K., Derry, S. J., Renkl, A., \& Wortham, D. (2000). Learning from examples: Instructional principles from the worked example research. Review of Educational Research, 70, 181-214.

Ausubel, D. P. (1964). Some psychological and educational limitations of learning by discovery. The Arithmetic Teacher, 11, 290-302.

Bandura, A. (1986). Social foundations of thought and action: A social cognitive theory. Englewoods Cliffs, NJ: Prentice Hall.

Bertsch, S., Pesta, B., Wiscott, R., \& McDaniel, M. (2007). The generation effect: A metaanalytic review. Memory and Cognition, 35, 201-210. doi: 10.3758/BF03193441

Chandler, P., \& Sweller, J. (1991). Cognitive load theory and the format of instruction. Cognition and Instruction, 8, 293-332.

Chi, M., Glaser, R., \& Rees, E. (1982). Expertise in problem solving. In R. Sternberg (Ed.), Advances in the psychology of human intelligence (pp. 7-75). Hillsdale, NJ: Erlbaum.

Cooper, G., \& Sweller, J. (1987). Effects of schema acquisition and rule automation on mathematical problem-solving transfer. Journal of Educational Psychology, 79, 347362.

Cowan, N. (2001). The magical number 4 in short-term memory: A reconsideration of mental storage capacity. Behavioral and Brain Sciences, 24, 87-185. 
Craig, R. (1956). Directed versus independent discovery of established relations. Journal of Educational Psychology, 47, 223-235.

Craik, F. I., \& Tulving, E. (1975). Depth of processing and the retention of words in episodic memory. Journal of experimental Psychology: general, 104(3), 268.

De Groot, A., \& Gobet, F. (1996). Perception and memory in chess: Heuristics of the professional eye. Assen, The Netherlands: Van Gorcum.

Ericsson, K. A., \& Kintsch, W. (1995). Long-term working memory. Psychological Review, $102,211-245$.

Griffith, D. (1976). The attentional demands of mnemonic control processes. Memory \& Cognition, 4(1), 103-108.

Jacoby, L. L., Craik, F. I., \& Begg, I. (1979). Effects of decision difficulty on recognition and recall. Journal of Verbal Learning and Verbal Behavior, 18(5), 585-600.

Kalyuga, S., Ayres, P., Chandler, P., \& Sweller, J. (2003). The expertise reversal effect. Educational Psychologist, 38, 23-31.

Kalyuga, S., Chandler, P., Tuovinen, J., \& Sweller, J. (2001). When problem solving is superior to studying worked examples. Journal of Educational Psychology, 93, 579588.

Kazemi, F., Ghoraishi, M. (2012). Comparison of Problem-based Learning Approach and traditional teaching on attitude, misconceptions and mathematics performance of University Students. Social and Behavioral Sciences, 46, 3852 - 3856.

Kirschner, P. A., Sweller, J., \& Clark, R. E. (2006). Why minimal guidance during instruction does not work: An analysis of the failure of constructivist, discovery, problem-based, experiential, and inquiry-based teaching. Educational Psychologist, $41,75-86$. 
Lai, P. \& Tang, C. (1999) Constraints affecting the implementation of a problem-based learning (PBL) Strategy in University Courses. Implementing Problem Based Learning Project: Proceedings of the First Asia Pacific Conference on Problem Base Learning (pp. 193-200).

Lee, H. (2013). Measuring cognitive load with electroencephalography and self-report: focus on the effect of English-medium learning for Korean students. Educational Psychology, (ahead-of-print), 1-11.

Mayer, R. (2004). Should there be a three-strikes rule against pure discovery learning? The case for guided methods of instruction. American Psychologist, 59, 14-19.

McElroy, L. A., \& Slamecka, N. J. (1982). Memorial consequences of generating nonwords: Implications for semantic-memory interpretations of the generation effect. Journal of Verbal Learning and Verbal Behavior, 21, 249-259.

McFarland Jr, C. E., Frey, T. J., \& Rhodes, D. D. (1980). Retrieval of internally versus externally generated words in episodic memory. Journal of Verbal Learning and Verbal Behavior, 19(2), 210-225.

McNamara, D., \& Healy, A. (2000). A procedural explanation of the generation effect for simple and difficult multiplication problems and answers. Journal of Memory and Language, 43, 652-679. doi: 10.1006/jmla.2000.2720

Miller, G. A. (1956). The magical number seven, plus or minus two: Some limits on our capacity for processing information. Psychological Review, 63, 81-97.

Paas, F. (1992). Training strategies for attaining transfer of problem-solving skill in statistics: A cognitive-load approach. Journal of Educational Psychology, 84, 429-434.

Paas, F., \& van Merrienboer, J. (1993). The efficiency of instructional conditions: An approach to combine mental-effort and performance measures. Human Factors, 35, 737-743. 
Paas, F., \& van Merrienboer, J. (1994). Variability of worked examples and transfer of geometrical problem-solving skills: A cognitive-load approach. Journal of Educational Psychology, 86, 122-133.

Peterson, L., \& Peterson, M. J. (1959). Short-term retention of individual verbal items. Journal of Experimental Psychology,58, 193-198.

Renkl, A. (1997). Learning from worked-out examples: A study on individual differences. Cognitive Science, 21, 1-29.

Schwonke, R., Renkl, A., Kreig, C., Wittwer, J., Aleven, V., \& Salden, R. (2009). The worked example-effect: Not an artefact of lousy control conditions. Computers in human behavior, 25, 258-266.

Shulman, L., \& Keisler, E. (Eds.). (1966). Learning by discovery: A critical appraisal. Chicago: Rand McNally.

Simon, H., \& Gilmartin, K. (1973). A simulation of memory for chess positions. Cognitive Psychology, 5, 29-46.

Slamecka, N. J., \& Katsaiti, L. T. (1987). The generation effect as an artifact of selective displaced rehearsal. Journal of Memory and Language, 26(6), 589-607.

Slamecka, N. J., \& Fevreiski, J. (1983). The generation effect when generation fails. Journal of Verbal Learning and Verbal Behavior, 22(2), 153-163.

Slamecka, N. J., \& Graf, P. (1978). The generation effect: Delineation of a phenomenon. Journal of Experimental Psychology: Human Learning and Memory, 4, 592-604.

Steffe, L., \& Gale, J. (Eds.) (1995). Constructivismin Education. Hillsdale, NJ: Lawrence Erlbaum.

Sweller, J. (1988). Cognitive load during problem solving: Effects on learning. Cognitive Science, 12, 257-285. 
Sweller, J. (1994). Cognitive load theory, learning difficulty, and instructional design. Learning and instruction, 4(4), 295-312.

Sweller, J. (2010). Element interactivity and intrinsic, extraneous and germane cognitive load. Educational Psychology Review, 22, 123-138.

Sweller, J., Ayres, P., \& Kalyuga, S. (2011). Cognitive load theory. New York: Springer.

Sweller, J., \& Chandler, P. (1994). Why some material is difficult to learn. Cognition and Instruction, 12, 185-233.

Sweller, J., \& Cooper, G. (1985). The use of worked examples as a substitute for problem solving in learning algebra. Cognition \& Instruction, 2, 59-89.

Sweller, J., \& Sweller, S. (2006). Natural information processing systems. Evolutionary Psychology, 4, 434-458.

Tindall-Ford, S., Chandler, P., \& Sweller, J. (1997). When two sensory modes are better than one. Journal of Experimental Psychology: Applied, 3, 257-287.

Tricot, A., \& Sweller, J. (2014). Domain-specific knowledge and why teaching generic skills does not work. Educational Psychology Review, 26, 265-283. doi: 10.1007/s10648013-9243-1

Tyler, S. W., Hertel, P. T., McCallum, M. C., \& Ellis, H. C. (1979). Cognitive effort and memory. Journal of Experimental Psychology: Human Learning and Memory, 5(6), 607.

Van Merriënboer, J. J. G., \& Sweller, J. (2005). Cognitive load theory and complex learning: Recent developments and future directions. Educational Psychology Review, 17, 147177. 